\title{
AN INFINITE-DIMENSIONAL HAMILTONIAN SYSTEM ON PROJECTIVE HILBERT SPACE
}

\author{
ANTHONY M. BLOCH
}

\begin{abstract}
We consider here the explicit integration of a Hamiltonian system on infinite-dimensional complex projective space. The Hamiltonian, which is the restriction of a linear functional to this projective space, arises in the problem of line fitting in complex Hilbert space (or, equivalently, the problem of functional approximation) or as the expectation value of a model quantum mechanical system. We formulate the system here as a Lax system with parameter, showing how this leads to an infinite set of conserved integrals associated with the problem and to an explicit formulation of the flow in action-angle form via an extension of some work of J. Moser. In addition, we find the algebraic curve naturally associated with the system.
\end{abstract}

Introduction. In recent years there has been a great deal of interest in integrable Hamiltonian systems in both finite and infinite dimensions. (See, for example, the work of Adler and van Moerbeke [2], Griffiths [11], McKean [16], Moser [18] and Mumford [19].) Most classical Hamiltonian systems of interest arise in the modelling of mechanical systems. In contrast, our Hamiltonian here arises as the Maximum Likelihood Estimation function for fitting a line to a set of data in a complex Hilbert space or, equivalently, as the distance between an $L^{2}$ function and its first Fourier component. Alternatively, the Hamiltonian may be viewed as the expectation value of a model quantum mechanical system where the Hamiltonian operator is compact. This expectation then generates the Heisenberg equations of motion as discussed in [8].

The work discussed here is a generalization to infinite dimensions and detailed discussion of the work announced in [4]. An announcement of the infinite-dimensional case was made in [7]. For the most part, the finite-dimensional case may be subsumed in the discussion of the infinite-dimensional case. One aspect that does not appear to carry over in a rigorous sense is the moment map argument for complete integrability (see $[\mathbf{4}, \mathbf{5}]$ ), although a convincing heuristic argument may be made.

As remarked in [4], the finite-dimensional system may be viewed as a system of the type dubbed by Adler and van Moerbeke [2] to be "spinning top or ellipsoid".

It is interesting that the analysis of the finite-dimensional system carries over so naturally to the infinite-dimensional context. In particular, aspects of the work of Moser on the integration of finite-dimensional integrable systems extend to this infinite-dimensional setting.

1. The Hamiltonian. A method of arriving at our Hamiltonian is to consider the problem of finding (and minimizing) the total perpendicular distance of a set

Received by the editors August 18, 1986.

1980 Mathematics Subject Classification (1985 Revision). Primary 34A05, 70H05, 53C57, 81C25.

Partially supported by a Horace A. Rockham faculty grant at the University of Michigan. 
of points in a Hilbert space to a given $d$-plane. This minimization problem we refer to as the Total Least Squares problem.

Let $\not$ be a complex separable Hilbert space. Let $\left(e_{j}\right)$ be an orthonormal basis for $\forall$ and suppose we have a countable sequence of points $\left(x_{i}\right)$ each given by

$$
x_{i}=\sum_{j} \lambda_{i j} e_{j}
$$

(We suppose for the purposes of statistical analysis that each $\lambda_{i j}$ is measured with observation error $\varepsilon_{i j}$, where the $\varepsilon_{i j}$ are $N(0,1)$ independent, although these assumptions may be weakened. We do not consider the statistical aspects of this problem in any detail here, but refer to [5 or 6].)

Now let $Q$ denote the orthogonal projection of $\not{H}$ onto a $d$-plane and let $P=I-Q$. Then, proceeding quite formally, we have

LEMMA 1.1. The total perpendicular distance of the points $x_{i}$ onto a d-plane in $\sharp$ is given by

$$
H(P)=\operatorname{Tr} C P=\operatorname{Tr} C-\operatorname{Tr} C Q=\operatorname{Tr} C-H_{0}(Q)
$$

where $Q$ is the rank $d$ projection and $C=\Lambda^{*} \Lambda$ where $\Lambda$ is the operator densely defined by the data matrix $\left(\lambda_{i j}\right)$.

ProOF. Let $\langle$,$\rangle denote the standard inner product on \mathcal{H}$. Then the distance of the $p$ points $x_{i}$ from the $d$-plane is

$$
\begin{aligned}
\sum_{i}\left\langle x_{i}, x_{i}\right\rangle-\left\langle Q x_{i}, Q x_{i}\right\rangle & =\sum_{i}\left\langle x_{i}, P x_{i}\right\rangle \\
& =\sum_{i, j, k} \bar{\lambda}_{i j} \lambda_{i k}\left\langle e_{j}, P e_{k}\right\rangle \\
& =\sum_{k, j} c_{k j} P_{j k} \quad \text { where } c_{k j}=\sum_{i} \bar{\lambda}_{i j} \lambda_{i k} .
\end{aligned}
$$

More precisely

LEMMA 1.2. $H(P)=\operatorname{Tr} C P$ is well defined when the operator defined by the data matrix is of Hilbert-Schmidt class or, equivalently, when $C$ is of trace class.

ProOF. For $\Lambda$ Hilbert-Schmidt class, $\Lambda^{*} \Lambda$ is Trace class. Since Trace class operators are a norm ideal in $B(\mathcal{H})$, the space of bounded operators on $\mathcal{H}, \operatorname{Tr} C P$ is well defined for all $P \in B(\mathcal{H})$.

Henceforth we assume $\Lambda$ to be Hilbert-Schmidt. (Actually, since we will confine our attention to $H_{0}(Q)=\operatorname{Tr} C Q$ for the Hamiltonian analysis, this assumption may be weakened to the assumption that $C$ is compact.)

In particular then, we may define the map $H: \operatorname{Grass}(d, \mathcal{H}) \rightarrow \mathbf{R}$ on the Grassmannian of $d$-planes in $\not$ via $H(P)=\operatorname{Tr} C P=\operatorname{Tr} C(I-Q)$.

We remark that the estimation problem on $\operatorname{Grass}(d, \mathcal{H})$ corresponds to a functional approximation problem for a set of $L^{2}$ functions by a set of $d$ basis vectors.

Suppose that we have a sequence of functions $f^{i}(\xi) \in L^{2}[a, b]$, say. Let $\left\{\psi_{j}(\xi)\right\}$, $j=1,2, \ldots$, be a complete set of orthogonal and normalized basis functions defined in the domain $[a, b]$. Any $f^{i}(\xi)$ may be expanded as

$$
f^{i}(\xi)=\sum_{i=1}^{\infty} \lambda_{i j} \psi_{j}(\xi)
$$


where

$$
\lambda_{i j}=\int_{a}^{b} \psi_{j}^{*}(\xi) f^{i}(\xi) d \xi
$$

and

$$
\sum_{j=1}^{\infty}\left|\lambda_{i j}\right|^{2}=1 .
$$

Then we consider minimizing the mean squared error

$$
\begin{aligned}
E\left(\left\{\psi_{i}\right\}, d\right) & =\sum_{i} \int_{a}^{b}\left|f^{i}(\xi)-\sum_{j=1}^{d} \lambda_{i j} \psi_{j}(\xi)\right|^{2} d \xi \\
& =\sum_{i} \sum_{j=d+1}\left|\lambda_{i j}\right|^{2}=1-\sum_{i} \sum_{j=1}^{d}\left|\lambda_{i j}\right|^{2}
\end{aligned}
$$

over the $\lambda_{i j}$ or, equivalently, over the $\psi_{j}$. This is clearly equivalent to the $d$-plane fitting problem. (See [20] for further details.)

As far as the Total Least Squares estimation problem is concerned, it is of great interest to determine the critical point structure of $H(P)=\operatorname{Tr} C P$. Since $\operatorname{Grass}(d, \mathcal{H})$ can be endowed with the structure of a complete Riemannian manifold in such a way that $H$ is smooth, it would seem that Morse theory would be the ideal tool to use. This turns out to be an interesting problem as $H(P)$ fails to obey condition (C) of Palais and Smale. Nonetheless the Morse-theoretic analysis can be carried out. For this analysis, see $[\mathbf{5}, \mathbf{9}]$. Here we restrict our attention to the Hamiltonian flow. As discussed briefly in $\S 4$ of the paper and in more detail in 5 and 6], the Hamiltonian flow does have statistical significance in the context of the Total Least Squares problem-it reflects the principal component structure of the data.

We remark also that the Total Least Squares estimate of the $d$-plane is the Maximum Likelihood Estimate under the assumption of normally distributed errors.

2. The symplectic structure and Hamiltonian flow. We now wish to endow the Hilbert manifold $\operatorname{Grass}(d, \mathcal{H})$ with a symplectic structure, and hence to find the Hamiltonian equations of motion corresponding to the Hamiltonian $H(P)=\operatorname{Tr} C P$ on $\operatorname{Grass}(d, \not{H})$.

Let $U(\not{H})$ be the Banach-Lie group of unitary operators on $\mathcal{H}$ and $u(\not{H})$ its Lie algebra of bounded skew-Hermitian operators. We define a finite rank orbit of $U(\mathscr{H})$ to be an adjoint orbit of finite rank operators in $u(\not H)$. The Grassmannians $G(d, \mathscr{H})$ are of this type (see $[\mathbf{1 4}$ and $\mathbf{1 5}]$ ). Now a Banach manifold $M$ is said to be symplectic if it possesses a closed (weakly) nondegenerate 2-form $\omega\left(\omega_{x}(\xi, \eta)=0 \forall \eta \in T_{x} M\right.$ implies $\xi=0$ ).

Further, making the identifications $Q \rightarrow i Q, C \rightarrow-i C$, whence we have $Q, C \in$ $u(n)$, we have

LEMMA 2.1. The finite rank orbits of $U(\not H)$ are symplectic. Hence the function $H_{0}(Q)=\operatorname{Tr} C Q$ (or $H(P)=\operatorname{Tr} C P$ ) may be regarded as a Hamiltonian on $\operatorname{Grass}(d, \mathscr{H})$, and the Hamiltonian equations of motion on $\operatorname{Grass}(d, \mathscr{H})$ for $H_{0}(Q)$ are given by the Lax equation $\dot{Q}=[Q, C]$. 
ProOF. The symplectic form can be defined as follows. Let $x$ be a point on the adjoint orbit and let $\xi$ and $\eta$ be tangent vectors to the orbit at $x$. Then we can write

$$
\xi=\left[x, a_{1}\right], \quad \eta=\left[x, a_{2}\right] \quad \text { some } a_{1}, a_{2} \in u(\not H) .
$$

Then define the "Kostant-Kirillov" form

$$
\omega_{x}(\xi, \eta)=\operatorname{Tr} x\left[a_{1}, a_{2}\right],
$$

which can be verified to have the requisite properties.

The Hamiltonian equations of motion then follow.

REMARKS. (1) $H_{0}(Q)$ differs from $H(P)$ by a constant. Hence, for the purposes of considering the dynamics, we consider $H_{0}(Q)$.

(2) In the case $Q$ is of rank 1 and the underlying manifold is $\mathbf{C P}^{\infty}$, complex projective space, $H_{0}(Q)$ can be regarded as the expectation value for the Hamiltonian operator $C$, and the equations of motion we have derived are the Heisenberg equations of motion. (See also [8].)

(3) For the remainder of the paper we consider the case where $Q$ is of rank $1-$ that is, the underlying manifold is complex projective space. The results generalize without essential change to the case $Q$ of higher rank by considering $Q$ to be a sum of rank 1 projections and hence $H_{0}(Q)$ to be a sum of Hamiltonians each with $Q$ of rank 1 .

3. Integrability. We now wish to discuss complete integrability of the Hamiltonian equations of motion $\dot{Q}=[Q, C]$ on complex projective space $\mathbf{C P}^{\infty}$. It is clear that the system of equations is integrable in terms of elementary functions. What we wish to do here is to generalize the ideas of complete integrability from finite dimensions - to find a complete set of commuting integrals for the flow, to find the algebraic curve associated with the system and to find the action-angle coordinates.

We note firstly that our Lax equations may be written as Lax equations with parameter. Let $\xi$ be a parameter. Then we have

LEMMA 3.1. The Lax equation $\dot{Q}=[Q, C]$ is equivalent to the Lax equation with parameter $(Q \dot{+} \xi C)=[Q+\xi C, C]$.

PROOF. The Lax equation with parameter reduces to the original Lax equation.

We have also that the Lax equation $\dot{Q}=[Q, C]$ is equivalent to the Lax equation $\dot{Q}=[Q, M], M=Q C+C Q$ and

LEMMA 3.2. The Lax equation $\dot{Q}=[Q, M]$ is equivalent to the Lax equation with parameter $(Q \dot{+} \xi C)=\left[Q+\xi C, M+\xi C^{2}\right]$.

We draw attention to this fact since in finite dimensions the Lax equations of Lemma 3.2 are, for $Q$ a generic (regular) element of $u(n)$, the generalized Euler (rigid body) equations on $U(n)$. (See [17], for example.)

Hence our problem here may be viewed as a degenerate case of the infinitedimensional rigid body problem on $U(\mathcal{H})$.

One method for deriving integrals of motion is to consider the functions $\operatorname{Tr}(Q+\xi C)^{k}, k=1,2, \ldots$, which are conserved on the flow of the Lax equation with parameter, since this flow is an isospectral deformation. 
Now, if the flow of our Lax equation is denoted by $Q(t)$, the flow of the corresponding Lax equation with parameter is given by $Q(t)+\xi C$. Hence the coefficients of $\xi$ in the expansion of $\operatorname{Tr}(Q+\xi C)^{k}$ will be conserved on the flow of the Lax equation without parameter. Explicit calculation then gives us

LEMMA 3.3. $\operatorname{Tr} C Q, \operatorname{Tr} C^{2} Q, \operatorname{Tr} C^{3} Q, \ldots$ are conserved under the flow of $H_{0}(Q)$ $=\operatorname{Tr} C Q$. Further, for regular $C$ ( $C$ having distinct eigenvalues), we have

LEMMA 3.4. The integrals $\operatorname{Tr} C Q, \operatorname{Tr} C^{2} Q, \ldots$ are in involution and independent on the adjoint orbit through $Q$.

ProOF. Involution follows since $C, C^{2}, \ldots$ commute and since we have a homomorphism from the Lie algebra $u(\not{K})$ to the set of vector fields on $\mathbf{C P}^{\infty}$ under the Poisson bracket.

Independence follows from the independence of the eigenvalues of $C$.

We remark that involution of the integrals may also be proved by the use of Lenard-type relations on the Lie algebra (as in Ratiu [20]).

Is Lemma 3.4 sufficient for complete integrability? Since we have one independent integral corresponding to every two real dimensions of the symplectic manifold $\mathbf{C P}^{\infty}$, we might argue quite heuristically that the system should be completely integrable. Indeed this is the definition of complete integrability given in McKean [16].

However, our argument for complete integrability need not rest on this as we can integrate the system explicitly. This we proceed to do in the next section via an analogue of the methods of Moser [18], in the process finding the algebraic curve associated with the system and the action-angle variables.

4. Explicit flow and the algebraic curve. Our main theorem is as follows.

THEOREM 4.1. Let $Q=\left(q_{i j}\right)$ be written $Q=z \otimes \bar{z}, z=\left(z_{1}, z_{2}, \ldots\right)$ where $z$ is a vector of norm 1 in the Hilbert space $\nLeftarrow, \sum_{i}\left|z_{i}\right|^{2}=1, z_{i}=x_{i}+i y_{i}$, and choose a basis such that the operator $C$ is diagonal, $C=\operatorname{diag}\left(c_{1}, c_{2}, \ldots\right)$. Then the algebraic curve associated with $H_{0}(Q)=\operatorname{Tr} C Q$ is given by

$$
\xi=\sum_{i} \frac{x_{i}^{2}+y_{i}^{2}}{\eta-c_{i}}
$$

and, via Moser's transformation, the Hamiltonian flow on $\mathbf{C P}^{\infty}$ with the KostantKirillov symplectic structure is shown to be given by

$$
q_{i j}(t)=\alpha_{i j} \exp \left(i\left(c_{j}-c_{i}\right) t+\beta_{i j}\right)
$$

where $\alpha_{i j}$ and $\beta_{i j}$ are constants. This flow is generated by the Hamiltonian $H=$ $\frac{1}{2} \sum_{i} c_{i}\left|z_{i}\right|^{2}$ on $\not{H}$ with the standard symplectic structure.

PROOF. For the proof we use an infinite-dimensional complex analogue of the work of Moser [18] in the case where a certain determinant, taken to be nonzero in [18], vanishes.

Firstly we need the Weinstein-Aronsjan formula.

We can describe a degenerate operator $T \in B\left(\mathcal{H}_{\mathbf{R}}\right)$, where $T$ is an operator on real Hilbert space $\nvdash_{\mathbf{R}}$ with inner product $\langle,\rangle_{\mathbf{R}}$, by two sets of linearly independent 
vectors $x_{1}, \ldots, x_{m}$ and $\rho_{1}, \ldots, \rho_{m}$ in the range of $T, R(T)$, where $m=\operatorname{rank} T$ :

$$
T v=\sum_{j=1}^{m} x_{i}\left\langle\rho_{i}, v\right\rangle_{\mathbf{R}}, \quad v \in \mathscr{H}_{\mathbf{R}} .
$$

Since $T$ is degenerate, we can $\operatorname{define} \operatorname{det}(1+T)$ by

$$
\operatorname{det}(1+T)=\operatorname{det}\left(1_{R}+T_{R}\right)
$$

where $T_{R}$ is the part of $T$ in $R=R(T)$ and $1_{R}$ is the identity operator in $R$. Then

$$
\operatorname{det}(1+T)=\operatorname{det}\left(\delta_{j k}+\left\langle\rho_{k}, x_{j}\right\rangle_{\mathbf{R}}\right) \text {. }
$$

Now with $S \in B(\mathcal{H}), T(S-\eta)^{-1}$ is degenerate and the Weinstein-Aronsjan determinant of the first kind (see [12])

$$
W(\eta, S, T)=\operatorname{det}\left(1+T(S-\eta)^{-1}\right)=\operatorname{det}\left((S+T-\eta)(S-\eta)^{-1}\right)
$$

is defined.

With

$$
\begin{gathered}
T=\sum_{j=1}^{m} x_{i}\left\langle\rho_{i}, \quad\right\rangle_{\mathbf{R}} \quad \text { and } \quad R(\eta)=(S-\eta)^{-1}, \\
W(\eta, S, T)=\operatorname{det}\left(\delta_{j k}+\left\langle R(\eta) x_{j}, \rho_{k}\right\rangle_{\mathbf{R}}\right),
\end{gathered}
$$

the Weinstein-Aronsjan formula.

Now specialize to rank two and $x_{1}=x, x_{2}=y, \rho_{1}=a x+b y, \rho_{2}=c x+d y$ where $a, b, c, d$ are constants, and let

$$
L=L(x, y)=C+a x \otimes x+b x \otimes y+c y \otimes x+d y \otimes y,
$$

a rank 2 perturbation of the Hermitian operator $C$. Now Moser takes (in finite dimensions) $\Delta=a d-b c \neq 0$. Here we differ, choosing $a=d=1, b=-c=i$, so $\Delta=0$.

Also, let $C$ be the matrix defined earlier. Letting

$$
\begin{aligned}
\Gamma_{x x} & =x \otimes x, \quad \Gamma_{y y}=y \otimes y, \quad \Gamma_{x y}=x \otimes y-y \otimes x \\
M_{z \bar{z}} & =z \otimes \bar{z}=Q, \quad \text { where } z=x+i y, \\
L & =L(x, y)=C+\Gamma_{x x}+\Gamma_{y y}+i \Gamma_{x y}=C+\Gamma_{z \bar{z}}=C+Q .
\end{aligned}
$$

We view $L$ as a complex rank one perturbation on the operator $C$ on our complex Hilbert space $\not$.

REMARK. We can consider the perturbation $C \xi+\Gamma_{z \bar{z}}$, but we note that $[Q+$ $C, C$ ] (without parameter $\xi$ ) gives a valid Lax equation for our problem, and for the work that follows (aside from finding the algebraic curve) it is more convenient to omit $\xi$.

Now the main result here is that the eigenvalues of $L$ are in involution with respect to the standard symplectic structure on complex Hilbert space

$$
\omega\left(z_{1}, z_{2}\right)=-\operatorname{Im}\left\langle z_{1}, z_{2}\right\rangle, \quad z_{1}, z_{2} \in \mathcal{K} .
$$

As in the finite-dimensional case of Moser [18], we will show that the symmetric functions of the eigenvalues of $L$ are in involution. 
Now the Weinstein-Aronsjan formula gives us

$$
\begin{aligned}
\operatorname{det}\left((\eta-L)(\eta-C)^{-1}\right) & =\operatorname{det}\left((\eta-C+Q)(\eta-C)^{-1}\right) \\
& =\operatorname{det}\left(1-W_{\eta}\right)=1-\operatorname{Tr} W \eta=1-\phi_{\eta}
\end{aligned}
$$

where

$$
\begin{aligned}
& W_{\eta}=\left[\begin{array}{ll}
Q_{\eta}(x) & Q_{\eta}(x, y) \\
Q_{\eta}(x, y) & Q_{\eta}(y)
\end{array}\right]\left[\begin{array}{cc}
1 & i \\
-i & 1
\end{array}\right], \\
& Q_{\eta}(x, y)=\left\langle R_{\eta} x, y\right\rangle_{\mathbf{R}}, \quad Q_{\eta}(x)=Q_{\eta}(x, x), \\
& R_{\eta}=(\eta I-C)^{-1}, \quad \phi_{n}=\operatorname{Tr} W_{\eta} .
\end{aligned}
$$

The involution result (after Moser [18, Theorem 1]) is

LEMMA 4.2. For any $\eta, \eta^{\prime}$ in the resolvent set of $C\left\{\phi_{\eta}, \phi_{\eta^{\prime}}\right\}=0$.

(The proof will be given as a corollary of Lemma 4.4.)

LEMMA 4.3. We can associate to our Lax equation the spectral curve

$$
\xi=\sum_{i} \frac{x_{i}^{2}+y_{i}^{2}}{\eta-c_{i}}
$$

ProOF. To obtain the spectral curve we consider

$$
\begin{aligned}
\operatorname{det}\left(\eta-\xi^{-1}\left(\xi C+\Gamma_{z \bar{z}}\right)\right) & =\operatorname{det}\left(\eta-C+\xi^{-1} \Gamma_{z \bar{z}}\right) \\
& \left.=\operatorname{det}(\eta-C)\left(1-\phi_{\eta}\right)=0 \quad \text { (see }[\mathbf{2}]\right)
\end{aligned}
$$

where

$$
\phi_{\eta}=\xi^{-1} Q_{\eta}(x)+\xi^{-1} Q_{\eta}(y)
$$

by the Weinstein-Aronsjan formula. Since $C$ is diagonal, we find

$$
\xi=\sum_{i} \frac{x_{i}^{2}+y_{i}^{2}}{\eta-c_{i}} .
$$

We note that the curve is well defined since it is equivalent to $\operatorname{Tr}(\eta I-C)^{-1} Q$, and $(\eta I-C)^{-1}$ is bounded while $Q$ is of Trace class.

We remark on the significance of the curve: since $Q+\xi C$ is undergoing an isospectral deformation, the spectral curve is independent of time. Hence we expect that the coefficients $x_{i}^{2}+y_{i}^{2}$ should be integrals for the flow, which we indeed see explicitly below.

What we wish to show now is that our Hamiltonian flow on complex projective space, with the Kirillov symplectic structure, is driven by a standard Hamiltonian flow on Hilbert space with the quite different natural symplectic structure on Hilbert space.

The idea is to extend the class of functions $\phi_{\eta}$. For a suitable analytic function $f(\eta)$ we let

$$
H(x, y)=\frac{1}{4 \pi_{i}} \int_{|\eta|=R} f(\eta) \phi_{\eta}(x, y) d \eta
$$

where $|\eta|=R$ contains the spectrum of $C$ (which we recall is compact). Setting $f\left(c_{j}\right)=\beta_{j}, \beta=\operatorname{diag}\left(\beta_{1}, \beta_{2}, \ldots\right)$ we get

$$
H=\frac{1}{2}\langle\beta x, x\rangle_{\mathbf{R}}+\frac{1}{2}\langle\beta y, y\rangle_{\mathbf{R}} .
$$


With $\beta_{j}=2 \delta_{j k}$ we get

$$
H(x, y)=G_{k}(x, y)=x_{k}^{2}+y_{k}^{2}=\left|z_{k}\right|^{2}
$$

which are in involution by Lemma 4.2 . Note that

$$
\phi(x, y)=\sum_{j} \frac{G_{j}(x, y)}{\eta-c_{j}}
$$

and

$$
H(x, y)=\frac{1}{2} \sum_{j} \beta_{j} G_{j}(x, y)=\frac{1}{2} \sum_{j} \beta_{j}\left|z_{j}\right|^{2} .
$$

Choosing $\beta_{j}=c_{j}$

$$
H=\frac{1}{2} \sum_{j=1} c_{j}\left(x_{j}^{2}+y_{j}^{2}\right)
$$

with equations of motion simply

$$
\dot{x}=\partial H / \partial y, \quad \dot{y}=-\partial H / \partial x .
$$

With $q_{i j}=(z \otimes \bar{z})_{i j}, z_{i}=x_{i}+i y_{i}$ we get the flow of Theorem 4.1. Further, we have the following crucial lemma (after Moser [18, Theorem 2]).

LEMMA 4.4. The vector field (B) with $H$ given by (A) defines an isospectral deformation of the matrix $L=Q+C$ given by $\dot{L}=[i C, L]$ or $(Q \dot{+} C)=[i C, Q+C]$.

REMARK. We have $i C$ here rather than $C$, as we assumed $C$ to be Hermitian.

We first show Lemma 4.2 is a corollary of Lemma 4.4. Since by Lemma 4.4, $L=Q+\xi C$ undergoes an isospectral deformation, any function of its eigenvalues $\lambda_{i}$ is constant along orbits.

Hence,

$$
1-\phi_{\eta}=\prod_{i} \frac{\eta-\lambda_{i}}{\eta-c_{i}}
$$

is an integral of motion for any $\eta$. Thus $(d / d t) \phi_{\eta}=\left\{\phi_{\eta}, H\right\}=0$. Taking $\beta_{j}=2 \delta_{j k}$ we get $H=G_{k}$. Therefore $\left\{\phi_{\eta}, G_{k}\right\}=0$ and

$$
\left\{\phi_{\eta}, \phi_{\eta^{\prime}}\right\}=\sum_{k}\left(\eta^{\prime}-c_{k}\right)^{-1}\left\{\phi_{\eta}, G_{k}\right\}=0 .
$$

ProOF OF LEMMA 4.4. Set $L=C+Q=C+S+R$ where $S$ and $R$ are the symmetric and antisymmetric parts of $Q$ respectively. Thus

$$
S=x \otimes x+y \otimes y, \quad R=i(x \otimes x-y \otimes y) .
$$

Now $H=\frac{1}{2}\langle C x, x\rangle_{\mathbf{R}}+\frac{1}{2}\langle C y, y\rangle_{\mathbf{R}}$. The differential equations for $X_{H}$ are

$$
\frac{d}{d t}\left(\begin{array}{l}
x \\
y
\end{array}\right)=\left(\begin{array}{cc}
0 & 1 \\
-1 & 0
\end{array}\right)\left(\begin{array}{l}
C x \\
C y
\end{array}\right)
$$

Hence $X_{H} R=i[C, S]$, that is

$$
X_{H} i(x \otimes y-y \otimes x)=i[C, x \otimes x+y \otimes y] .
$$


This holds since

$$
\begin{aligned}
\frac{d}{d t}(x \otimes y-y \otimes x) & =\frac{d}{d t}(x \otimes y)-\frac{d}{d t}(y \otimes x) \\
& =C y \otimes y-x \otimes x C+C x \otimes x-y \otimes y C .
\end{aligned}
$$

Similarly, for $X=x \otimes x+y \otimes y$

$$
X_{H} S=-[C, x \otimes y-y \otimes x] .
$$

Hence

$$
\begin{aligned}
X_{H} L=X_{H}(R+S) & =i[C, x \otimes x+y \otimes y]-[C, x \otimes y-y \otimes x] \\
& =i[C, x \otimes x+y \otimes y]+i[C, i x \otimes y-i y \otimes x] \\
& =[i C, C+x \otimes x+i x \otimes y-i y \otimes x+y \otimes y] \\
& =[i C, L] .
\end{aligned}
$$

This concludes the proof of Lemma 4.4 and hence of Theorem 4.1.

5. Concluding remarks. (1) We note that in the finite-dimensional case our system fits into the list of completely integrable systems of "spinning top and ellipsoid type" (see [2]) in the following sense.

We have the matrix $L(x, y)$ (see $\S 4$ ) taking the forms

(i) $a \xi+\Gamma_{x y} \quad(n$-dimensional free rigid body problem),

(ii) $a \xi^{2}+\xi \Gamma_{x y}-\Gamma_{x x}$ (geodesic flow on the ellipsoid; Neumann's problem),

(iii) $a \xi^{2}+\xi \Gamma_{x y}+(x \otimes y+y \otimes x-a) \quad$ (central force problem on ellipsoid).

To this list we have added

(iv) $a \xi+\Gamma_{x x}+\Gamma_{y y}+i \Gamma_{x y}$

(line-fitting problem, model quantum mechanical system).

We note that our system is in some sense the most "symmetric" or most basic of these systems as reflected by the fact that the associated algebraic curve is rational rather than hyperelliptic, and that the flow can be expressed in terms of elementary functions rather than $\theta$-functions.

(2) As discussed in [5 and 6], when the Hamiltonian is viewed as the Total Least Squares Estimation function, the Hamiltonian flow has an interesting statistical interpretation. In fact the Hamiltonian flow reflects the principal component structure of the data. From $\S 4$ we see that under the Hamiltonian flow each matrix entry $q_{i j}$ of $Q$ oscillates with frequency equal to the modulus of the difference $c_{j}-c_{i}$ between the eigenvalues $c_{j}$ and $c_{i}$ of $C$. In terms of principal component analysis (see $[\mathbf{1 3}]$ ), these frequencies are the differences between variances of the principal components of the data.

(3) As discussed in [8], the Moser transformation of $\S 4$ is the transformation from the Heisenberg picture to the Schroedinger picture for a model quantum mechanical system where the Hamiltonian operator $C\left(H_{\mathrm{op}}\right.$ in the notation of [8]) is compact. Thus the Heisenberg and Schroedinger representations involve two different symplectic structures. Note also that our Lax formulation of the Hamiltonian equations of motion gives us an infinite set of commuting integrals for the Heisenberg equations. 
(4) It would be very interesting to extend the analysis here to the case of a more complicated operator $C-C$ being noncompact/unbounded. This is of particular interest for quantum mechanical systems. Work on this case is in progress.

ACKNOWLEDEGMENT. I would like to thank my advisor at Harvard, Professor Chris Byrnes, for providing the inspiration for this work. I would also like to thank Professor Roger Brockett for his useful comments, and the referee for his comments and suggestions.

\section{REFERENCES}

1. R. A. Abraham and J. E. Marsden, Foundations of mechanics, Benjamin/Cummings, 1978.

2. M. Adler and P. van Moerbeke, Completely integrable systems, Euclidean Lie algebras and curves, Adv. in Math. 38 (1980), 318-379.

3. V. I. Arnold, Mathematical methods of classical mechanics, Springer-Verlag, 1978.

4. A. M. Bloch, A completely integrable Hamiltonian system associated with line fitting in complex vector spaces, Bull. Amer. Math. Soc. 12 (1985), 250-254.

5. _ Completely integrable Hamiltonian systems and total least squares estimation, $\mathrm{Ph}$. D. Thesis, Harvard Univ., 1985.

6. __ Estimation, principal components and Hamiltonian systems, Systems Control Lett. 6 (1985), 1-15.

7. _ , Total least squares estimation in infinite dimensions and completely integrable Hamiltonian systems, Proc. 7th Internat. Conf. on the Mathematical Theory of Networks and Systems, Stockholm, 1985 (to appear).

8. __ An infinite-dimensional classical integrable system and the Heisenberg and Schroedinger representations, Phys. Lett. 116A (1986), 353-355.

9. A. M. Bloch and C. I. Byrnes, An infinite-dimensional variational problem arising in estimation theory, Algebraic and Geometric Methods in Nonlinear Control Theory (M. Fliess and M. Hazewinkel, Eds.), Reidel, 1986.

10. C. I. Byrnes and J. C. Willems, Least squares estimation, linear programming and momentum, preprint.

11. P. A. Griffiths, Linearizing flows and a cohomological interpretation of the Lax equation, preprint.

12. T. Kato, Perturbation theory for linear operators, Springer-Verlag, 1966.

13. D. G. Kendall, Multivariate analysis, Macmillan, 1975.

14. P. de la Harpe, Classical Banach-Lie algebras and Banach-Lie groups of operators in Hilbert space, Lecture Notes in Math., vol. 285, Springer-Verlag, 1972.

15. B. Maissen, Lie-Gruppen mit Banachräumen als Parameterraume, Acta Math. 108 (1962), 229-270.

16. H. P. McKean, Integrable systems and algebraic curves, Lecture Notes in Math., vol. 755, Springer-Verlag, 1978, pp. 83-200.

17. A. S. Mischenko and A. T. Fomenko, Integrability of Euler equations on semisimple Lie algebras, Select. Math. Soviet. 2 (1982), 207-292.

18. J. Moser, The geometry of quadrics and spectral theory, Proceedings of the Chern Symposium 1979, Springer-Verlag, 1980.

19. D. Mumford, Tata lectures on theta. II, Birkhäuser, 1984.

20. T. Ratiu, The motion of the free $n$-dimensional rigid body, Indiana Univ. Math. J. 29 (1980), $609-629$.

21. S. Watanabe, Karhunen-Loève expansion and factor analysis, theoretical remarks and applications, Transactions of the 4th Prague Conference on Information Theory, Publ. House Czechoslovak Acad. Sci., Prague, 1965.

Department of Mathematics, University of Michigan, Ann Arbor, Michigan 